\section{Foxgl Foxg1}

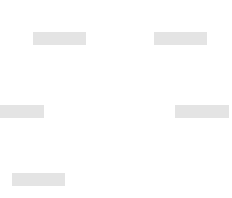

\section{Cerebellum DNasel HS Peaks Rep 1 from ENCODE/UW}

\section{Cerebrum DNasel HS Peaks Rep 1 from ENCODE/UW}

Whole Brain Adult 8 Weeks DNasel HS Peaks Rep 1 from ENCODE/UW

Whole Brain Embryonic Day 14.5 DNasel HS Peaks Rep 1 from ENCODE/UW

Cerebellum 8w H3K4me3 Histone Mods by ChIP-seq Peaks from ENCODE/LICR Cerebellum 8w H3K27ac Histone Mods by ChIP-seq Peaks from ENCODE/LICR Cerebellum 8w H3K27me3 Histone Mods by ChIP-seq Peaks from ENCODE/LICR

Cortex 8w H3K4me3 Histone Mods by ChIP-seq Peaks from ENCODE/LICR

Cortex 8w H3K27ac Histone Mods by ChIP-seq Peaks from ENCODE/LICR

Liver 8w H3K4me3 Histone Mods by ChIP-seq Peaks from ENCODE/LICR Liver 8w H3K27ac Histone Mods by ChIP-seg Peaks from ENCODE/LICR Liver 8w H3K27me3 Histone Mods by ChIP-seq Peaks from ENCODE/LICR 